\title{
ONLINE COURSE REGISTRATION APPLICATION AT EDEN EVERYDAY ENGLISH BOGOR
}

\author{
Dinda Ayu Muthia ${ }^{1)}$, Yetrivo Efendy ${ }^{2)}$ \\ ${ }^{1,2}$ Sistem Informasi, Universitas Bina Sarana Informatika \\ ${ }^{2}$ Sistem Informasi, Universitas Bina Sarana Informatika \\ ${ }^{1}$ Kp. Sidamukti RT/RW 005/006 No. 3, Depok \\ ${ }^{2}$ Jl. Paledang Lebak Sari RT/RW 01/03, Bogor

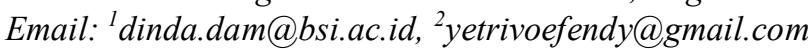

\begin{abstract}
The course is one of the education provided outside formal (non-formal) schools to develop personal abilities and skills. Eden Everyday English Course Institute is an Institution located in Bogor. In this institution, the registration process for students is done manually by filling in the registration form provided by the admin. Eden Everyday English Course Institute is one of the courses where the number of applicants increases every year, this causes the administration department cannot manage the registration properly and feels overwhelmed so that it is not effective. With the increase in the number of students registering each year, it would be better if a web-based system was created, so that it could simultaneously serve as a medium for promoting the course institution. This research purpose is to build a web-based application to help the registration process for new students in Eden Everyday English Course Institute using the waterfall method. Many studies, especially in the field of information system development, use the Waterfall method. This application improves the quality of information and the efficiency of the use of online course registration.
\end{abstract}

Keyword: Online, Course, Regisration, Web, Application

\begin{abstract}
Abstrak
Kursus merupakan salah satu pendidikan yang diberikan di luar sekolah formal (non formal) untuk mengembangkan kemampuan dan keterampilan pribadi. Eden Everyday English Course Institute adalah sebuah Institusi yang berlokasi di Bogor. Di institusi ini, proses pendaftaran mahasiswa dilakukan secara manual dengan mengisi formulir pendaftaran yang disediakan oleh admin. Eden Everyday English Course Institute merupakan salah satu lembaga kursus yang jumlah pendaftarnya semakin meningkat setiap tahunnya, hal ini menyebabkan bagian administrasi tidak dapat mengelola pendaftaran dengan baik dan merasa kewalahan sehingga tidak efektif. Dengan bertambahnya jumlah mahasiswa yang mendaftar tiap tahunnya, akan lebih baik jika dibuat sistem registrasi berbasis web, sehing ga sekaligus bisa menjadi media promosi lembaga kursus. Tujuan penelitian ini adalah membuat aplikasi yang berbasis web untuk membantu proses pendaftaran mahasiswa baru di Eden Everyday English Course Institute dengan menggunakan metode waterfall. Banyak penelitian, khususnya di bidang pengembangan sistem informasi, menggunakan metode Waterfall. Aplikasi ini meningkatkan kualitas informasi dan efisiensi pelaksanaan pendaftaran kursus online.
\end{abstract}

Kata Kunci: Online, Kursus, Pendaftaran, Web, Aplikasi

\section{Introduction}

English has become a mandatory language for anyone who wants to be successful globally. The increasing flow of people and cross-border communication places English as the social language on the world stage. It is as if an agreement has been reached, that every interaction in the international world will use English [1].

English is the language used to communicate throughout the world. Today everyone must learn and master English in order to keep up with the rapid development of globalization. This was done because English is indispensable in any aspect [2]. Second language is usually the official language or language that is socially dominant and necessary in education, work and other basic needs [3].

This research took place in Eden Everyday English Course Institute, Bogor. In this institution, the admission process for students is done manually by filling in the registration form provided by the admin. Eden Everyday English Course Institute is one of the courses where the number of applicants increases every year, this causes the administration department cannot manage everything properly and feels overwhelmed so that it is not effective. With the increase in the number of students registering each year, it would be better if a web-based registration 
system was created, so that it could simultaneously serve as a medium for promoting the course institution.

The software development method used in this research is Waterfall method. Many studies, especially in the field of information system development, use the Waterfall method, such as a research conducted by Tri Hartati, namely the design of an electronic information system for new student registration at language and professional education institute (LBPP) LIA Pramuka, Jakarta [4], online student admission application at SMK Al-Basyariah Bojong Gede [5], and online application for student admission in SMK Negeri 3 Yogyakarta [6].

This research has a purpose that is to create a webbased application to help the registration process for new students in Eden Everyday English Course Institute using the waterfall method.

\section{Method}

Based on Ian Sommerville [7], Waterfall model have five principal stages, namely as follows:

1. Requirements definition

2. System and software design

3. Implementation and unit testing

4. Integration and system testing

5. Operation and maintenance.

This research only conducts 3 phase of Waterfall model, namely:

1) Requirements definition

Observations have been conducted in Eden Everyday English to analyze the needs in the course registration system. This is conducted so that when a computerized system is created, all system requirements are met.

2) System and software design

The author designed an online course registration system with the relationship between the entities in the system described in the Entity Relationship Diagram and Logical Record Structure. As for making object-oriented system design using UML (Unified Modeling Language).

3) Implementation and unit testing

At this stage, online course registration application is made using the PHP programming language. In addition, unit testing is also conducted through Black Box Testing.

\section{Results and Discussion}

A. Functional Requirements

In this online registration course application there are users who have access to the web, namely: Administrator and Student. Both users have different requirements, such as:

1. Administrator Requirements:

a. Manage course package data

b. Manage schedule

c. Manage Student data
2. Student Requrements:

a. Register

b. Payment Confirmation

B. Database Design

The relationship between some entity is described in Entity Relationship Diagram. Figure 1 illustrated the diagram.

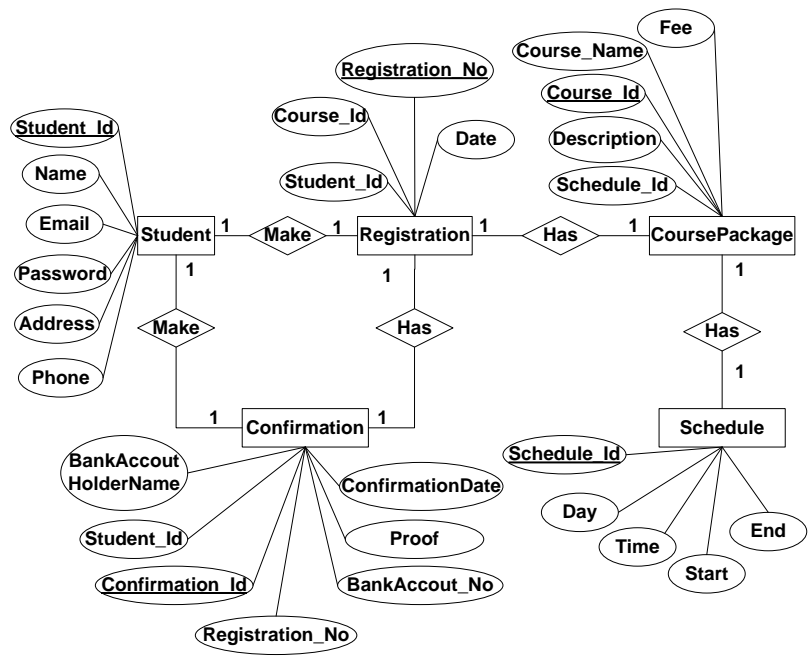

Figure 1. Entity Relationship Diagram

Figure 2 depicts logical record structure of the database design.

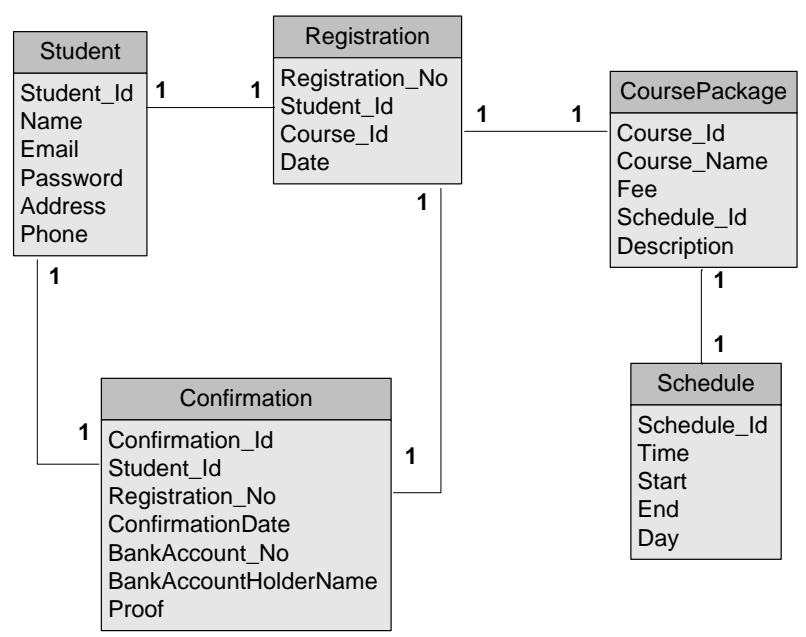

Figure 2. Logical Record Structure

C. Use Case Diagram

The design of use case diagram of the proposed system has been depicted in Figure 3. 


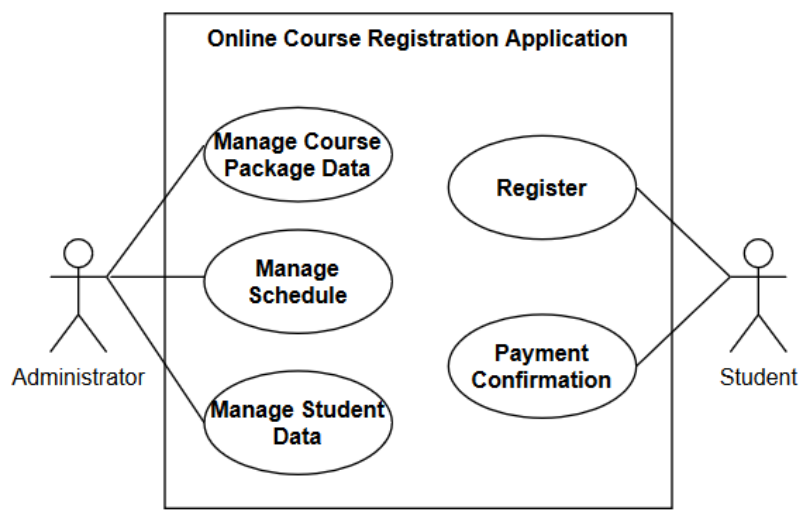

Figure 3. Use Case Diagram

D. Activity Diagram

This diagram is made based on each use case. Based on "Manage Schedule" use case, activity diagram is depicted in Figure 4.

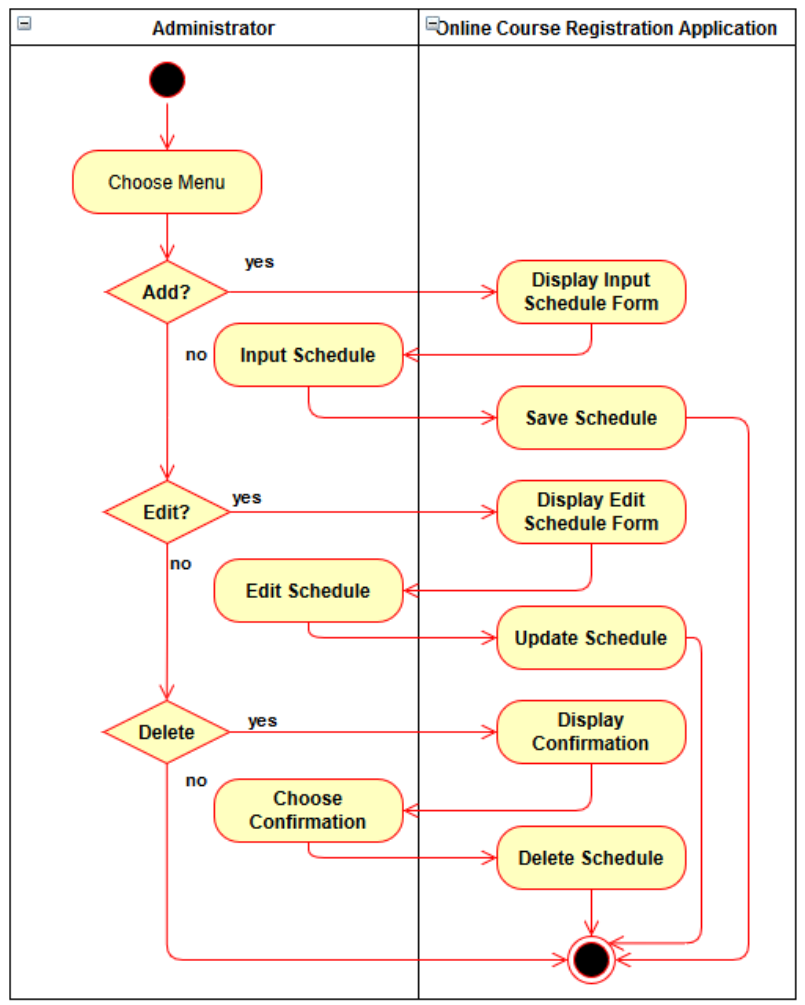

Figure 4. Activity Diagram

E. Implementation

The web of proposed system can be seen in these Figures below. Administrator log in page can be seen in Figure 5.

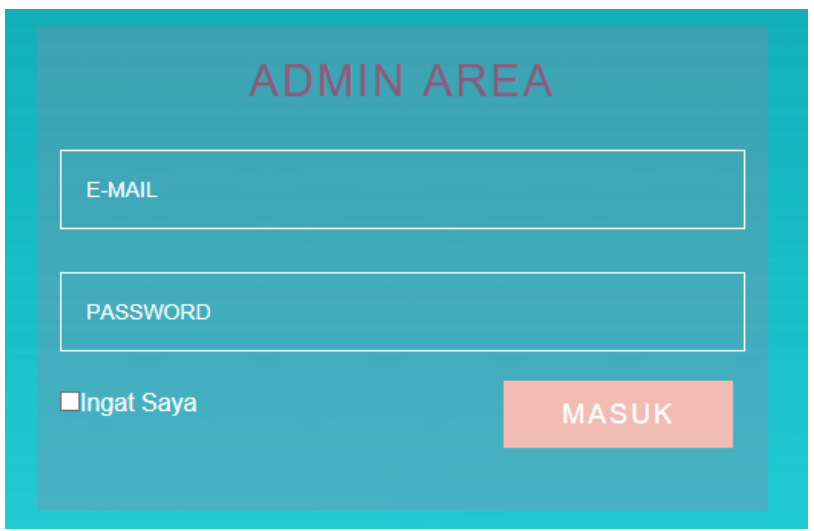

Figure 5. Administrator Log In Page

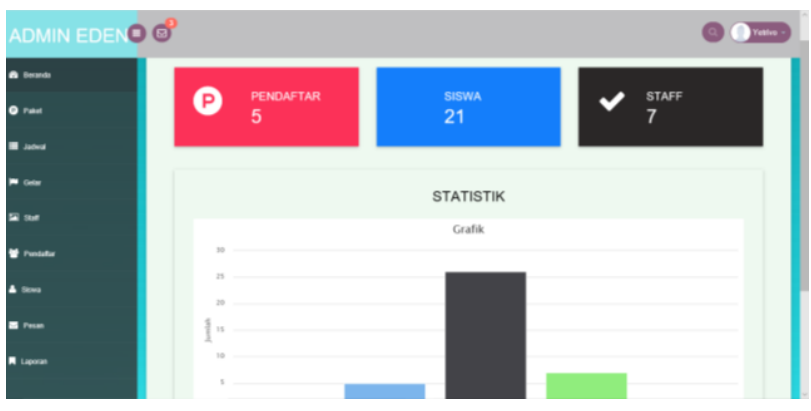

Figure 6. Administrator Home Page

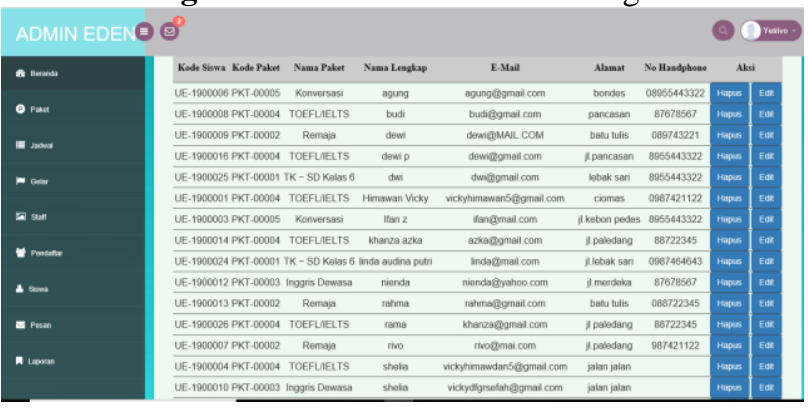

Figure 7. Student Data Page

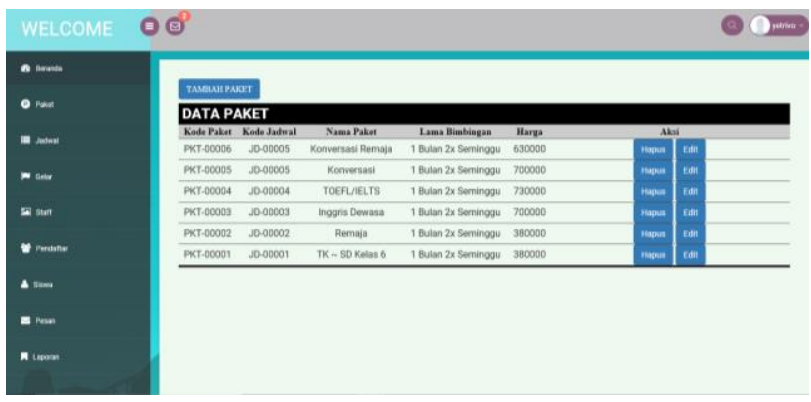

Figure 8. Course Package Data Page 


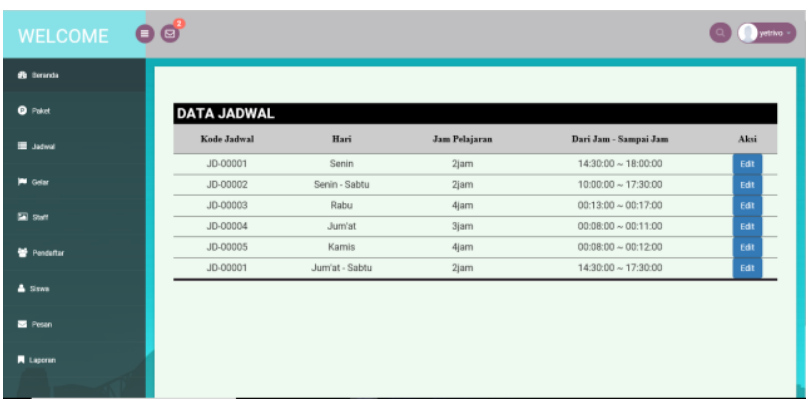

Figure 9. Schedule Page

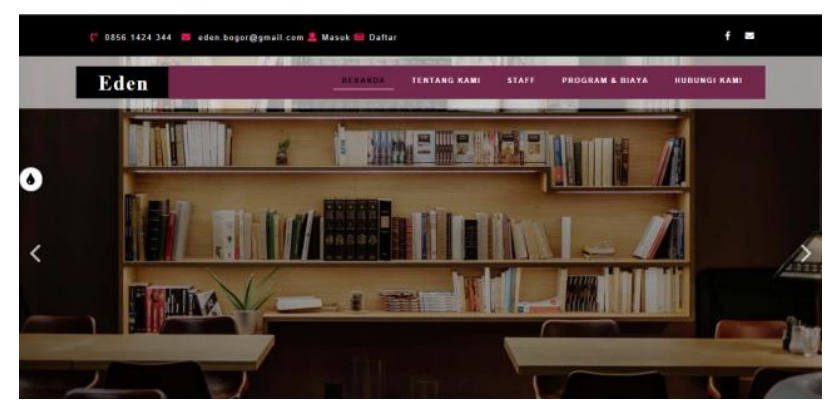

Figure 10. Home Page

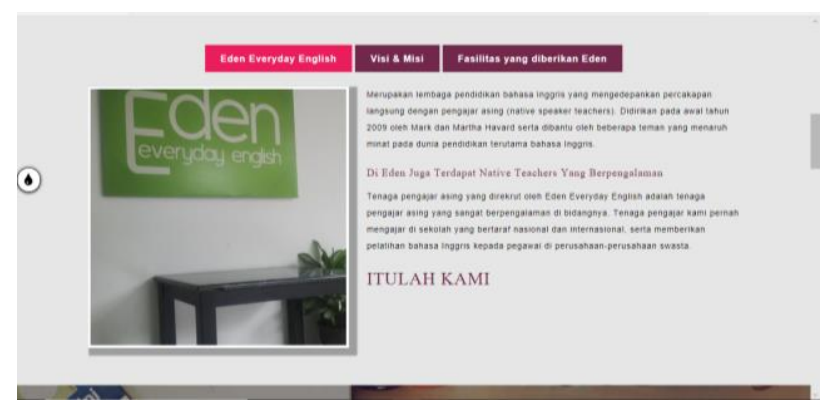

Figure 11. About Us Page

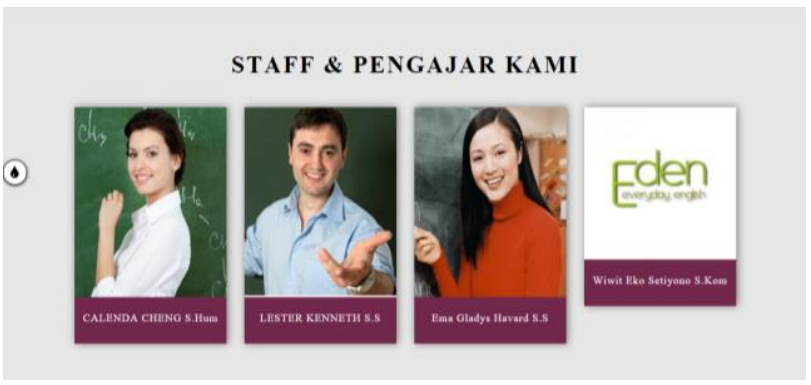

Figure 12. Our Staff

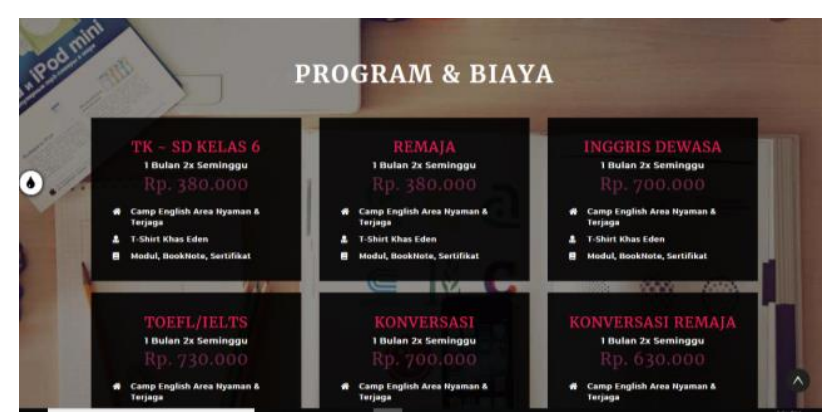

Figure 13. Course Package Page

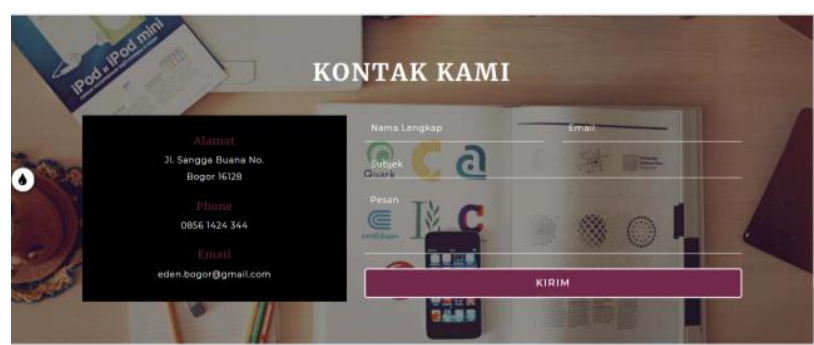

Figure 14. Contact Us Page

F. Black-box Testing

Table 1 depicted the process of black-box testing on administrator's log in page.

Table 1. Black-Box Testing on Administrator's

\begin{tabular}{|c|c|c|c|c|c|}
\hline \multicolumn{6}{|c|}{ Log In Page } \\
\hline No & $\begin{array}{c}\text { Testing } \\
\text { scenario }\end{array}$ & $\begin{array}{l}\text { Test } \\
\text { Case }\end{array}$ & $\begin{array}{l}\text { Expected } \\
\text { result }\end{array}$ & Result & $\begin{array}{l}\text { Conclu } \\
\text { sion }\end{array}$ \\
\hline 1. & $\begin{array}{l}\text { Email } \\
\text { and } \\
\text { password } \\
\text { are } \\
\text { not filled } \\
\text { then } \\
\text { click } \\
\text { the login } \\
\text { button }\end{array}$ & $\begin{array}{l}\text { Email } \\
\text { (empt } \\
\text { y) } \\
\text { Pass } \\
\text { word } \\
\text { (empt } \\
\text { y) }\end{array}$ & $\begin{array}{l}\text { The } \\
\text { system } \\
\text { will deny } \\
\text { user } \\
\text { access } \\
\text { and } \\
\text { display } \\
\text { "Please } \\
\text { fill out } \\
\text { this } \\
\text { field" }\end{array}$ & $\begin{array}{c}\text { Compat } \\
\text { ible to } \\
\text { expecta } \\
\text { tions }\end{array}$ & Valid \\
\hline 2. & $\begin{array}{l}\text { Email is } \\
\text { filled in } \\
\text { and the } \\
\text { password } \\
\text { is not } \\
\text { filled in } \\
\text { or blank } \\
\text { then } \\
\text { click the } \\
\text { login } \\
\text { button }\end{array}$ & $\begin{array}{l}\text { Email } \\
\text { (ara } \\
\text { @.gm } \\
\text { ail.co } \\
\text { m) } \\
\text { Pass } \\
\text { word } \\
\text { (empt } \\
\text { y) }\end{array}$ & $\begin{array}{l}\text { The } \\
\text { system } \\
\text { will deny } \\
\text { user } \\
\text { access } \\
\text { and } \\
\text { display } \\
\text { "Please } \\
\text { fill out } \\
\text { this } \\
\text { field" }\end{array}$ & $\begin{array}{c}\text { Compat } \\
\text { ible to } \\
\text { expecta } \\
\text { tions }\end{array}$ & Valid \\
\hline 3. & $\begin{array}{l}\text { Email on } \\
\text { textfield } \\
\text { is not } \\
\text { blank } \\
\text { and } \\
\text { password } \\
\text { is filled } \\
\text { in, and } \\
\text { then } \\
\text { click the } \\
\text { login } \\
\text { button }\end{array}$ & $\begin{array}{l}\text { Email } \\
\text { (empt } \\
\text { y) } \\
\text { Pass } \\
\text { word } \\
\text { (1234 } \\
\text { 5) }\end{array}$ & $\begin{array}{l}\text { The } \\
\text { system } \\
\text { will deny } \\
\text { user } \\
\text { access } \\
\text { and } \\
\text { display } \\
\text { "Login } \\
\text { Failed" }\end{array}$ & $\begin{array}{c}\text { Compat } \\
\text { ible to } \\
\text { expecta } \\
\text { tions }\end{array}$ & Valid \\
\hline
\end{tabular}




\begin{tabular}{|c|c|c|c|c|c|}
\hline 4. & $\begin{array}{l}\text { Fill in } \\
\text { one of } \\
\text { the } \\
\text { wrong } \\
\text { condition } \\
\mathrm{s} \text { in the } \\
\text { email or } \\
\text { password } \\
\text { then } \\
\text { click the } \\
\text { login } \\
\text { button }\end{array}$ & $\begin{array}{l}\text { Email } \\
\text { (ara } \\
\text { @gm } \\
\text { ail.co } \\
\text { m) } \\
\text { Pass } \\
\text { word } \\
(0000 \\
0)\end{array}$ & $\begin{array}{l}\text { The } \\
\text { system } \\
\text { will deny } \\
\text { user } \\
\text { access } \\
\text { and } \\
\text { display } \\
\text { "Login } \\
\text { Failed" }\end{array}$ & $\begin{array}{l}\text { Compat } \\
\text { ible to } \\
\text { expecta } \\
\text { tions }\end{array}$ & Valid \\
\hline 5. & $\begin{array}{l}\text { Fill in } \\
\text { the email } \\
\text { textfield } \\
\text { and } \\
\text { password } \\
\text { textfield } \\
\text { with the } \\
\text { correct } \\
\text { data, and } \\
\text { then } \\
\text { click the } \\
\text { login } \\
\text { button }\end{array}$ & $\begin{array}{l}\text { Email } \\
\text { (ara } \\
\text { @.gm } \\
\text { ail.co } \\
\text { m) } \\
\text { Pass } \\
\text { word } \\
(1234 \\
5)\end{array}$ & $\begin{array}{l}\text { The web } \\
\text { system } \\
\text { accepts } \\
\text { login } \\
\text { access } \\
\text { and } \\
\text { displays } \\
\text { "successf } \\
\text { ul login" } \\
\text { then } \\
\text { immediat } \\
\text { ely } \\
\text { displays } \\
\text { user info }\end{array}$ & $\begin{array}{l}\text { Compat } \\
\text { ible to } \\
\text { expecta } \\
\text { tions }\end{array}$ & Valid \\
\hline
\end{tabular}

Table 2. Black Box Testing on Registration Form

\begin{tabular}{|c|c|c|c|c|c|}
\hline No & $\begin{array}{c}\text { Testing } \\
\text { scenario }\end{array}$ & $\begin{array}{l}\text { Test } \\
\text { Case }\end{array}$ & $\begin{array}{l}\text { Expected } \\
\text { result }\end{array}$ & Result & $\begin{array}{c}\text { Conclu } \\
\text { sion }\end{array}$ \\
\hline 1. & $\begin{array}{l}\text { All fields } \\
\text { are } \\
\text { empty } \\
\text { then } \\
\text { click the } \\
\text { register } \\
\text { button }\end{array}$ & $\begin{array}{l}\text { Name } \\
: \\
\text { (empt } \\
\text { y) } \\
\text { Email } \\
: \\
\text { (empt } \\
\text { y) } \\
\text { Pass } \\
\text { word: } \\
\text { (empt } \\
\text { y) } \\
\text { Addr } \\
\text { ess: } \\
\text { (empt } \\
\text { y) } \\
\text { Phon } \\
\text { e: } \\
\text { (empt } \\
\text { y) }\end{array}$ & $\begin{array}{l}\text { The } \\
\text { system } \\
\text { will } \\
\text { display } \\
\text { "Please } \\
\text { fill out } \\
\text { this } \\
\text { field" }\end{array}$ & $\begin{array}{l}\text { Compat } \\
\text { ible to } \\
\text { expecta } \\
\text { tions }\end{array}$ & Valid \\
\hline 2. & $\begin{array}{l}\text { All fields } \\
\text { are filled, } \\
\text { except } \\
\text { Name } \\
\text { field then } \\
\text { click the } \\
\text { register } \\
\text { button }\end{array}$ & $\begin{array}{l}\text { Name } \\
: \\
\text { (empt } \\
\text { y) } \\
\text { Anot } \\
\text { her } \\
\text { fields } \\
: \\
\text { (filled }\end{array}$ & $\begin{array}{l}\text { The } \\
\text { system } \\
\text { will } \\
\text { display } \\
\text { "Please } \\
\text { fill out } \\
\text { this } \\
\text { field" }\end{array}$ & $\begin{array}{c}\text { Compat } \\
\text { ible to } \\
\text { expecta } \\
\text { tions }\end{array}$ & Valid \\
\hline
\end{tabular}

\begin{tabular}{|c|c|c|c|c|c|}
\hline & & $\begin{array}{l}\text { with } \\
\text { valid } \\
\text { data) }\end{array}$ & & & \\
\hline 3. & $\begin{array}{l}\text { All fields } \\
\text { are filled, } \\
\text { except } \\
\text { Email } \\
\text { field then } \\
\text { click the } \\
\text { register } \\
\text { button }\end{array}$ & $\begin{array}{l}\text { Email } \\
: \\
\text { (empt } \\
\text { y) } \\
\text { Anot } \\
\text { her } \\
\text { fields } \\
: \\
\text { (filled } \\
\text { with } \\
\text { valid } \\
\text { data) }\end{array}$ & $\begin{array}{l}\text { The } \\
\text { system } \\
\text { will } \\
\text { display } \\
\text { "Please } \\
\text { fill out } \\
\text { this } \\
\text { field" }\end{array}$ & $\begin{array}{l}\text { Compat } \\
\text { ible to } \\
\text { expecta } \\
\text { tions }\end{array}$ & Valid \\
\hline 4. & $\begin{array}{l}\text { All fields } \\
\text { are filled, } \\
\text { except } \\
\text { Password } \\
\text { field then } \\
\text { click the } \\
\text { register } \\
\text { button }\end{array}$ & $\begin{array}{l}\text { Pass } \\
\text { word: } \\
\text { (empt } \\
\text { y) } \\
\text { Anot } \\
\text { her } \\
\text { fields } \\
: \\
\text { (filled } \\
\text { with } \\
\text { valid } \\
\text { data) }\end{array}$ & $\begin{array}{l}\text { The } \\
\text { system } \\
\text { will } \\
\text { display } \\
\text { "Please } \\
\text { fill out } \\
\text { this } \\
\text { field" }\end{array}$ & $\begin{array}{l}\text { Compat } \\
\text { ible to } \\
\text { expecta } \\
\text { tions }\end{array}$ & Valid \\
\hline 5. & $\begin{array}{l}\text { All fields } \\
\text { are filled, } \\
\text { except } \\
\text { Address } \\
\text { field then } \\
\text { click the } \\
\text { register } \\
\text { button }\end{array}$ & $\begin{array}{l}\text { Addr } \\
\text { ess: } \\
\text { (empt } \\
\text { y) } \\
\text { Anot } \\
\text { her } \\
\text { fields } \\
: \\
\text { (filled } \\
\text { with } \\
\text { valid } \\
\text { data) }\end{array}$ & $\begin{array}{l}\text { The } \\
\text { system } \\
\text { will } \\
\text { display } \\
\text { "Please } \\
\text { fill out } \\
\text { this } \\
\text { field" }\end{array}$ & $\begin{array}{l}\text { Compat } \\
\text { ible to } \\
\text { expecta } \\
\text { tions }\end{array}$ & Valid \\
\hline 6. & $\begin{array}{l}\text { All fields } \\
\text { are filled, } \\
\text { except } \\
\text { Phone } \\
\text { field then } \\
\text { click the } \\
\text { register } \\
\text { button }\end{array}$ & $\begin{array}{l}\text { Phon } \\
\text { e: } \\
\text { (empt } \\
\text { y) } \\
\text { Anot } \\
\text { her } \\
\text { fields } \\
: \\
\text { (filled } \\
\text { with } \\
\text { valid } \\
\text { data) }\end{array}$ & $\begin{array}{l}\text { The } \\
\text { system } \\
\text { will } \\
\text { display } \\
\text { "Please } \\
\text { fill out } \\
\text { this } \\
\text { field" }\end{array}$ & $\begin{array}{c}\text { Compat } \\
\text { ible to } \\
\text { expecta } \\
\text { tions }\end{array}$ & Valid \\
\hline
\end{tabular}

\section{Conclusion}

Based on the discussion that the author has discussed previously, the problem of Course Registration at Eden Everyday English Bogor takes a long time and is less effective. With this proposed system design, it is 
expected to minimize existing deficiencies. So from the system design made the author can conclude as follows:

1. With this online course registration application, it is hoped that Eden Everyday English will make it easier to produce new registrant data reports.

2. This application can make it easier for users to get information, especially prospective new registrants who will register so that it is more effective without having to queue for a long time.

3. This application facilitates registration and payment data processing and minimizes errors.

\section{References}

[1] D. L. Fithri, "Analisa dan Perancangan ELearning Pembelajaran Grammer untuk Meningkatkan Potensi Siswa," J. Simetris, vol. 5, no. 1, pp. 67-74, 2014.

[2] B. W. Sari, E. Utami, and H. Al Fatta, "Penerapan Konsep Gamification Pada Pembelajaran Tenses Bahasa Inggris Berbasis Web," J. Ilm. SISFOTENIKA, vol. 5, no. 2, pp.
155-166, 2015.

[3] M. Saville-Troike, Introducing Second Language Acquisition. Cambridge: Cambridge University Press, 2006.

[4] T. Hartati, "Perancangan Sistem Informasi Electronic New Student Registration Studi Kasus Lembaga Bahasa dan Pendidikan Profesional (LBPP) LIA Pramuka Jakarta," J. Tek. Inform., vol. 12, no. 2, pp. 193-200, 2019.

[5] D. A. Muthia, A. Ramadhani, A. Kurniawan, and R. Irfansyah, "Online Student Admission Application at SMK Al-Basyariah Bojong Gede," J. Sink., vol. 3, no. 2, pp. 1-6, 2019.

[6] N. H. Cahyana, E. Y. Prasetyo, and H. Himawan, "APLIKASI PENERIMAAN SISWA BARU BERBASIS WEB," J. Telemat., vol. 10, no. 1, pp. 1-8, 2013.

[7] I. Sommerville, Software Engineering Ninth Edition. Pearson Education, Inc, 2011. 